\title{
Multiwavelength analysis as a probe of accretion and radiative processes in LINERs
}

\author{
George Younes ${ }^{1}$ and Delphine Porquet ${ }^{2}$ \\ ${ }^{1}$ NSSTC, 320 Sparkman Drive, Huntsville, AL 35805, USA \\ email: gyounes@usra.edu \\ ${ }^{2}$ Observatoire Astronomique de Strasbourg, CNRS, UMR7550, \\ 11 rue de l'Universit, 67000, Strasbourg, France \\ email: delphine.porquet@astro.unistra.fr
}

\begin{abstract}
We study the multiwavelength properties of an optically selected sample of Low Ionization Nuclear Emission-line Regions (LINERs), in an attempt to determine the accretion mechanism powering their central engine. We show how their X-ray spectral characteristics, and their spectral energy distribution compare to luminous AGN, and briefly discuss their connection to their less massive counter-parts galactic black-hole X-ray binaries.
\end{abstract}

Keywords. AGN, LINERs, accretion, accretion disks

\section{Introduction}

Low luminosity active galactic nuclei (LLAGN) have grown to become one of the most interesting facets of modern AGN astrophysics. The challenge that these fascinating objects present lies in understanding their unusual dimness, leading to a better comprehension of the faint end of the AGN luminosity function. Low Ionization Nuclear Emission-line Regions (LINERs) make up 1/3 of these LLAGN and mainly populate the nearby universe. These LINERs exhibit bolometric luminosities at least two orders of magnitude lower than classical luminous AGN (Seyferts and quasars). At such low luminosities, the optically thick geometrically thin accretion disk powering luminous AGN, is believed to switch to a geometrically thin, optically thick, radiatively inefficient accretion flow (Narayan \& McClintock 2008). The switch occurs below a critical mass accretion rate $\dot{M}$, for which LINERs clearly belongs (Ho 2009), where the density of the disk becomes too low for radiative cooling to be effective and most of the energy in the flow is advected beyond the $\mathrm{BH}$ event horizon and/or convected away in the form of outflows/jet (Narayan \& Yi 1995; Blandford \& Begelman 1999). In the following we give the results on the X-ray and multiwavelength properties of an optically selected sample of LINERs showing the definite detection of broad H $\alpha$ line (LINER 1s, Ho et al. 1997). We discuss these results in the context of accretion in LINERs specifically and LLAGN in general.

\section{Results and discussion}

The X-ray spectra of all LINER 1s in our sample are fit with an absorbed power-law, sometimes including the contribution of a thermal component at low energies $(<1.5 \mathrm{keV})$. They all lacked the $\mathrm{Fe} \mathrm{K} \alpha$ line at $6.4 \mathrm{keV}$ and the soft excess detected in most luminous AGN. Moreover, only one of the LINER 1s showed significant variability on time-scales $<1$ day, a popular property among Seyfert and quasars. Finally, we found that the power-law photon index is anticorrelated with the Eddington ratio for LINER 1s, i.e., 
the spectra are hardening with increasing $L_{\mathrm{bol}} / L_{\mathrm{Edd}}$ (Younes et al. 2011). The opposite is found for luminous AGN, with their X-ray spectra softening with increasing Eddington ratio (Porquet al. 2004). We note that X-ray binaries in their low/hard state share this last similar characteristic with LINERs (Wu \& Gu 2008).

The lack of an Fe line in all LINER 1s might indicate the absence of an optically thick reflecting medium, thought to be the inner-edge of a thin accretion disk in luminous AGN (we note, though, that only $30 \%$ of luminous type 1 AGN show relativistic Fe K $\alpha$ lines, Nandra et al. 2007). The rare short time-scale variability could be the result of a larger X-ray emitting region compared to luminous AGN. Both properties fit the RIAF picture very well. Additionally, the anticorrelation between $\Gamma$ and $L_{\mathrm{bol}} / L_{\mathrm{Edd}}$ is easily explained in the RIAF context, where the X-ray emission is the result of inverse-Compton scattering of the synchrotron radiation in the flow. The increase in $L_{\mathrm{bol}} / L_{\mathrm{Edd}}$, roughly equivalent to an increase in $\dot{M}$, will result in more photons being scattered to higher energies by the hot electrons in the flow, causing the X-ray spectrum to harden.

We have built the spectral energy distribution of LINER 1s in our sample with simultaneous UV to X-ray data, mainly coming from the XMM-Newton telescope, eliminating the long time-scale variability in these two bands that could bias our conclusions (Younes et al. 2010; Younes et al. 2012). We confirmed previous results on LINER SEDs that came from random non-simultaneous observations; mainly, (1) at a given X-ray luminosity, the radio emission of LINER $1 \mathrm{~s}$ is comparable to the radio emission of radio-loud quasars, (2) the UV big blue bump is absent with an almost flat UV to X-ray flux ratio, $\alpha_{\text {ox }}$, and (3) their bolometric luminosity derived from their SEDs is at least two orders of magnitude lower than luminous AGN. We found, for the first time for LINER $1 \mathrm{~s}$, that $\alpha_{\text {ox }}$ is anticorrelated with Eddington ratio (Younes et al. 2012), in contrast to the positive relation found for luminous AGN (Lusso et al. 2010). Such an anticorrelation for LINERs has been predicted based on X-ray binaries in their low/hard state (Sobolewska et al. 2011). Such an anticorrelation could also be explained in the RIAF context where the $\mathrm{X}$-ray luminosity decreases faster than the UV luminosity with decreasing accretion rate (Xu 2011).

\section{References}

Blandford, R. D. \& Begelman, M. C. 1999, M.N.R.A.S., 303, L1

Ho, L. C. 2009, Ap.J., 699, 626

Ho, L. C., Filippenko, A. V., Sargent, W. L. W., \& Peng, C. Y. 1997, ApJS, 112, 391

Lusso, E., Comastri, A., Vignali, C., et al. 2010, A\&A, 512, A34+

Nandra, K., O’Neill, P. M., George, I. M., \& Reeves, J. N. 2007, M.N.R.A.S., 382, 194

Narayan, R. \& McClintock, J. E. 2008, New A Rev., 51, 733

Narayan, R. \& Yi, I. 1995, Ap.J., 444, 231

Porquet, D., Reeves, J. N., O’Brien, P., \& Brinkmann, W. 2004, A\& $A, 422,85$

Sobolewska, M. A., Siemiginowska, A., \& Gierliński, M. 2011, M.N.R.A.S., 413, 2259

Wu, Q. \& Gu, M. 2008, Ap.J., 682, 212

Younes, G., Porquet, D., Sabra, B., Reeves, J. N., \& Grosso, N. 2012, A\&A, 539, A104+

Younes, G., Porquet, D., Sabra, B., \& Reeves, J. N. 2011, A\&SA, 530, A149+

Younes, G., Porquet, D., Sabra, B., et al. 2010, A\&A, 517, A33+ 